\title{
Safety and efficacy of biodegradable nasopore nasal packing in functional endoscopic sinus surgery
}

\author{
Abstract \\ Background: Nasal packing is often used after functional endoscopic sinus surgery \\ (FESS). Various \\ non-absorbable agents are used but these packs can be uncomfortable and cause pain and \\ bleeding on removal. Increasingly, biodegradable nasal packs are being used and have \\ shown promising effects on post-operative bleeding and healing after FESS.
}

Aim: We evaluate the safety and efficacy of biodegradable Nasopore $($ (polyurethane glycol) in FESS, particularly in the day-case setting.

Methods: A retrospective chart review of 100 consecutive patients receiving Nasopore following FESS.

Results: Our patients had chronic rhinosinusitis not responding to medical therapy and the extent of the surgery was determined by the extent of disease. Procedures performed in addition to FESS included septoplasty, trimming of the inferior turbinates and polypectomy. Approximately half of patients were discharged home the same day and post-operative complications were minimal. Only two patients developed post-operative bleeding, and four reported post-operative sinus infection requiring oral antibiotics. One patient developed minor intranasal adhesions of no functional significance.

Conclusions: Nasopore is a safe and effective option after FESS. It is associated with minimal discomfort, no pain on removal and few post-operative complications. Nasopore is useful in achieving day-case FESS.

Keywords: epistaxis, sinusitis, nasal polyps, rhinitis, haemostatic technique, paranasal sinus Disease, endoscopy, surgery induced tissue adhesions
Volume I Issue 2 - 2014

\author{
Goldie S,' Shakeel M,' Trinidade A, ${ }^{2}$ \\ Vallamkondu V,' Ram B,' Ah-See KW' \\ 'Department of Otolaryngology, Aberdeen Royal Infirmary, UK \\ 2Department of Otolaryngology, Norfolk \& Norwich University \\ Hospital, UK
}

\begin{abstract}
Correspondence: Muhammad Shakeel, Department of Otolaryngology, Ward 45, Aberdeen Royal Infirmary, Foresterhill Road, Aberdeen AB252ZN, UK, Tel 01224552100 , Fax
\end{abstract} 0I224554569, Email drshakeel@doctors.org.uk

Received: December 26, 2014 | Published: December 30, 2014
Abbreviations: FESS, functional endoscopic sinus surgery; CRS, chronic rhinosinusitis; SPF, synthetic polyurethane foam

\section{Introduction}

Chronic rhinosinusitis (CRS) is a significant cause of morbidity affecting between 6.9 and $27.1 \%$ of the European population. ${ }^{1}$ Most commonly patients complain of facial congestion, nasal obstruction, rhinorrhoea, postnasal discharge and hyposmia with resultant poor quality of life. Medical treatment of CRS involves topical steroids, nasal douching, short term nasal decongestants and long term antibiotics in resistant cases. However, when medical therapy fails, surgical intervention in the form of functional endoscopic sinus surgery (FESS) may become necessary. This is the gold standard in the management of medically refractory chronic rhinosinusitis.

Common post-operative complications of FESS include bleeding and infection. Over the first few weeks, the patients can develop crusting and adhesions between the middle turbinate and the lateral nasal wall. The latter can lead to further sinus blockage and a recurrence of symptoms of CRS. There is no agreed management for the prevention of these complications in the literature. Among the recommended methods, nasal packing has been suggested to achieve haemostasis, and to prevent middle meatal adhesions when placed as a spacer lateral to the middle turbinate.

Nasal packs can be broadly divided into two groups: non- absorbable and biodegradable. Non absorbable agents include petroleum jelly impregnated gauze strips and expandable polyvinyl acetate. These packs are uncomfortable to patients as removal can be painful and can cause some bleeding. ${ }^{2}$ More recently, biodegradable packs have become available and include gelatine matrix, esterified hyaluronic acid, polyethylene glycol, oxidised regenerated cellulose and gelatine. ${ }^{3}$ Several studies have demonstrated the effectiveness of these materials by reducing or eliminating removal and preventing complications such as postoperative bleeding and adhesion..$^{4-6}$ There is great debate about whether the nose should be packed and if so, which nasal pack performs best. Conflicting studies suggest decreased and increased rates of postoperative complications such as bleeding, ${ }^{7,8}$ crusting or scarring and synechia formation ${ }^{8}$ when compared to no nasal packing. This conflict is further compounded by a lack of randomised, comparative and prospective studies between packing agents.

Recently there has also been an increasing drive in our Trust to perform FESS as a day case procedure. To facilitate this we utilise Nasopore ${ }^{\circledR}$ (Stryker Canada, Hamilton, Ontario, Canada), a biodegradable, synthetic polyurethane foam, after FESS. This avoids the need for removal and allows patients to return home after their operation, assuming their recovery has been uneventful. In this study we aim to assess the effectiveness of Nasopore in assisting with the goal of day case sinus surgery and to investigate the incidence of postoperative complications. 


\section{Materials and methods}

The study was carried out at the Aberdeen Royal Infirmary, a tertiary referral centre in the North East of Scotland with a catchment area of 550,000 people, including those from the offshore island communities of Orkney and Shetland.

One hundred consecutive patients suffering from symptoms of CRS underwent FESS by a single surgeon over a 2-year period between January 2010 and January 2012. FESS was performed either in isolation or in conjunction with septoplasty, trimming of inferior turbinates, endoscopic nasal polypectomy or a combination of these. All patients received treatment by the same surgeon and had Nasopore placed into the middle meatus at the end of the operation. Patients were excluded if they underwent surgery for sinonasal malignancy.

Clinic letters of patients undergoing endoscopic sinus surgery were retrospectively reviewed. Along with demographics, data were collected on date of admission to the hospital, date of operation, date of discharge from the hospital, geographical location of patients' home, pre-operative symptomatology, type of operation and post-operative progress focusing on complications and subsequent management

All patients received at least one pre-operative appointment where they were assessed for symptoms of anosmia, hyposmia, nasal obstruction, rhinorrhoea, purulent discharge, epistaxis, postnasal drip, catarrh, facial pain, headaches, cough and snoring. All operations were carried out under general anaesthetic. The nasal preparation was performed using xylometazoline nasal drops. The root of middle turbinate was infiltrated with $2 \%$ lidocaine with 1:80,000 adrenaline. The extent of the operation was decided based on the patients' symptoms, clinical and radiological findings. Intraoperatively, complications such as excessive bleeding, and breach of skull base were noted.

Most patients were routinely followed up but some failed to attend their post-operative review appointments while few patients were discharged after the operation with no intended follow up. Those who were followed up usually attended the clinic within the first 2 months after the operation. Late post-operative complications such as synechia formation and excessive granulation tissue were assessed with rigid nasendoscopy at follow-up. As granulation tissue is a normal process of healing, excessive granulation tissue was defined as

that involving more than $10 \%$ of the middle meatus. If residual Nasopore was identified this was removed with suction. Symptom improvement and any new symptoms were also noted.

Data were collected and analysed using Microsoft Excel. Ethical approval was granted for this retrospective review by the clinical effectiveness department of Aberdeen Royal Infirmary.

\section{Results}

Of the 100 patients, 55 were male and there was a mean age of 50 years (range 19-81). All patients had standard symptoms of CRS but $90 \%$ in the study had a detailed description of symptoms. Of those, described symptoms were as follows: nasal obstruction (78\%), facial pain $(33 \%)$, hyposmia $(20 \%)$, anosmia $(11 \%)$, post nasal drip $(28 \%)$, headaches (17\%) and rhinorrhoea (16\%) (Figure 1).

Out of 100 patients, the most common operations performed were FESS with septoplasty, trimming of inferior turbinates and polypectomy (23\%), FESS with septoplasty and trimming of inferior turbinates (22\%) and FESS with polypectomy (19\%) (Figure 2). Forty-nine percent of the patients were discharged home on the same day. Factors resulting in overnight admissions included distance of residence from the hospital, in particular residents of Orkney and Shetland (Table 1) and the timing of surgery, with those undergoing surgery in the afternoon being more likely to stay overnight (Table 2).

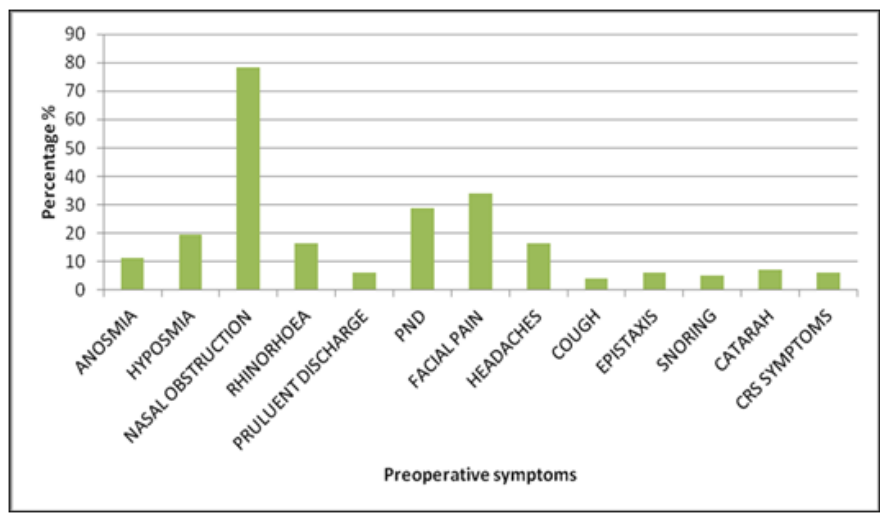

Figure I The symptomatology of those undergoing FESS for chronic rhinosinusitis. PND= post nasal discharge. The most common symptom described is nasal obstruction followed by facial pain, disruption of smell (hyposmia and anosmia) and facial pain.

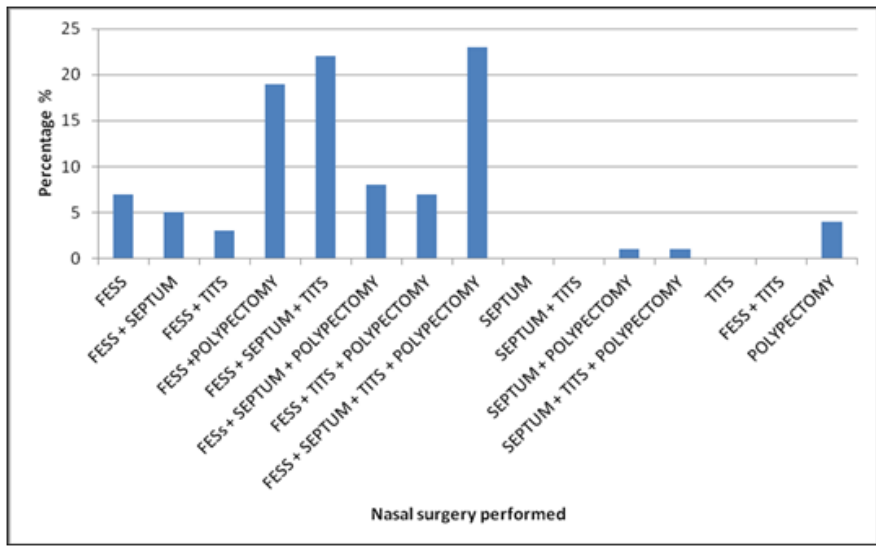

Figure 2 The distribution of operations carried out for chronic rhinosinusitis between January 2010 and January 2012 .

FESS, functional endoscopic sinus surgery; Septum, septoplasty; TITS, trimming of inferior turbinates; polypectomy, nasal polypectomy

Table I Number of nights patients stayed depending on distance from hospital. $48 \%$ of those who stayed on the mainland stayed overnight compared with $83 \%$ who stayed on a peripheral island

\begin{tabular}{llcll}
\hline \multicolumn{5}{c}{$\begin{array}{l}\text { Patient nights } \\
\text { spent in hospital }\end{array}$} \\
\hline Distance (miles) & 0 & 1 & Total & $\begin{array}{l}\text { Percentage staying } \\
\text { overnight \% }\end{array}$ \\
\hline$<15$ & 25 & 21 & 46 & $45.7 \%$ \\
\hline $15-30$ & 3 & 10 & 13 & $76.9 \%$ \\
\hline$>30$ & 20 & 15 & 35 & $42.9 \%$ \\
\hline Peripheral island & 1 & 5 & 6 & $83.3 \%$ \\
\hline
\end{tabular}


Table 2 Time of operation vs. duration of stay. Those receiving an operation in the afternoon were $20 \%$ more likely to stay overnight

\section{Patient nights \\ spent in hospital}

\begin{tabular}{lccll}
\hline Operation time & 0 & 1 & Total & $\begin{array}{l}\text { Percentage group } \\
\text { staying overnight } \%\end{array}$ \\
\hline Morning & 30 & 21 & 51 & $41.2 \%$ \\
\hline Afternoon & 19 & 30 & 49 & $61.2 \%$ \\
\hline
\end{tabular}

After surgery $75 \%$ of patients were followed up, $6 \%$ failed to attend and $9 \%$ were discharged. Of the patients available for follow up, all reported subjective benefit from surgery. Observed post-operative complications included crusting $(6 \%)$, infection $(4 \%)$, epistaxis $(2 \%)$ synechia (1\%), dural exposure (1\%) and surgical emphysema of the eyelid $(1 \%)$. With respect to post-operative bleeding, one patient required Merocel packing immediately post-operatively due to ooze from his trimmed inferior turbinates. One patient developed bleeding on post-operative Day 6 from septal granulation tissue and this was successfully managed by additional Nasopore packing in the clinic. Four patients reported post-operative sinus infection requiring oral antibiotics by their general practitioner. One patient was found to have minor adhesions between the middle turbinate and the septum but these were asymptomatic and therefore not deemed of any clinical significance. No nasal packing related complications were reported by the patients.

\section{Discussion}

Available since 1969, biodegradable packs and gels have undergone significant advances in recent years., ${ }^{9,10}$ Those that have been reported on in patient trials include gelatin, fibrin glue, microporus polysaccharide beads, oxidised regenerated cellulose, hyaluronic acid, synthetic polyurethane foam (SPF) and polyethylene glycol. Whilst the mechanisms of action many of these have been extensively studied, relatively little has been reported about Nasopore, with no studies investigating its absorption kinetics or mechanical properties. $^{10}$

There is great variation in patient expectations of endoscopic sinus surgery. Most underestimate the amount of epistaxis postoperatively which can cause significant anxiety. ${ }^{11,12}$ Pain upon pack removal is a common observation in many studies and significantly affects patient's willingness to reuse a particular pack. ${ }^{2}$ Finding the most suitable pack to optimise patient experience is therefore desirable.

Absorbable packs have been observed to reduce patient pain upon removal. In one study, patient perspectives were examined in those packed with SPF (synthetic polyurethane foam; Polyganics BV, Goningen, Netherlands) and Merocel (expandable polyvinyl acetate; Metronic Xiomed, Jacksonville, FL, USA). No significant differences in pain, pressure, nasal obstruction, anterior rhinorrhoea, postnasal drip, dysphagia and sleep disturbance were found. However, upon removal, the SPF population noted significantly less pain $(7.4 \pm 1.0 \mathrm{cf}$ $3.3 \pm 1.1$ ) and as a consequence there was greater patient satisfaction and willingness to reuse a pack in the SPF group. ${ }^{2}$ The authors surmised that as pack removal was conducted on the second postoperative day, SPF had degraded to a greater extent and was removed by suction avoiding irritation of the inflamed epithelium. Merocel retained its consistency and volume necessitating its removal by forceps. This caused greater irritation of the inflamed epithelium and dislodged clots causing further pain and bleeding. ${ }^{2}$ Conversely, Nasopore was compared with Merocel in a study of 30 adults for postoperative complications such as pain, pressure, swelling, nasal blockage, bleeding and packing removal. There were no significant differences between the packs although this may have been a consequence of low study numbers. ${ }^{13}$

Some surgeons believe that in addition to discomfort, nasal packing causes unnecessary complications. Orlandi et al., ${ }^{14}$ advocated the use of selective packing in a retrospective review of 165 patients. They selectively packed 19 patients finding none of the patients had a major postoperative bleed. ${ }^{14}$ With respect to biodegradable packs, there is little evidence to support this hypothesis. In fact some evidence suggests that they may be protective Wee et al., ${ }^{8}$ compared Gelfoam (Pharmica \& Upjohn Co., NY, USA), a gelatin foam, with no packing in those undergoing FESS. Subjective symptoms such as nasal obstruction, postnasal drip and bleeding were assessed both directly after surgery and two weeks later. Interestingly, there was no significant difference between patient scores in either group. ${ }^{15}$ In another study, SPF powder demonstrated no significant differences between pain, discharge and obstruction when compared to a 'no treatment' arm during recovery from FESS. ${ }^{12}$ Finally, Floseal (a bovine collagen/gelatin matrix which is combined with bovine thrombin prior to use to form a haemostatic agent; Baxter International Inc, Deerfield, IL, USA), when compared to no packing, demonstrated a significant reduction in pain postoperatively $\left(\mathrm{X}^{2}=4.88, \mathrm{p}=0.027\right) .{ }^{8}$ Although our study did not formally asses post-operative pain, we demonstrated that Nasopore packs can safely be left in situ in $100 \%$ of cases, hence eradicating potentially painful removal (Figure 3).

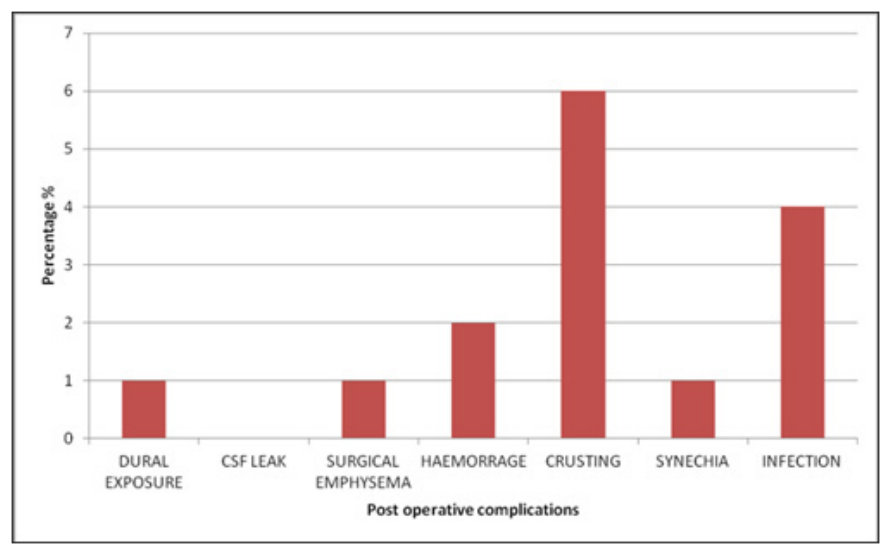

Figure 3 Post operative complications encountered after surgery. The most common of which includes crusting (6\%), Infection (4\%) and Hameorrage (2\%).

The risk of significant haemorrhage following FESS is very small ${ }^{16}$ but there is often a small degree of epistaxis which can lead to patient anxiety and negatively affect patient perception of recovery. ${ }^{12}$ Trauma during pack removal is one of the causes. Upon removing nasal packing on the second post-operative day, Kim et al., ${ }^{2}$ observed that only $18.8 \%$ of those packed with SPF following FESS had epistaxis requiring intervention compared with $81 \%$ of a Merocel group. ${ }^{2}$ The effect is similar when biodegradable nasal packs are left in situ. When Floseal and Merocel were compared, the incidence of postoperative epistaxis was significantly higher in the Merocel group (27.81\%; $\mathrm{p}<0.001 \%$ ) which was directly attributable to the physical act of pack removal. ${ }^{17}$ Absorbable packs thus reduce epistaxis both when either removed or left in situ when compared to Merocel packs. In our study, 99\% of patients were treated with Nasopore alone and consequently did not require formal pack removal. 
Synechiae may lead to failure of FESS and has an incidence of approximately $8 \% .{ }^{4,18}$ These fibrin-like structures, which are produced when two inflamed and denuded mucosal surfaces adhere, often result in disruption of the sinus drainage pathways leading to disease recurrence. Increased crusting and scarring is likely to promote this process. ${ }^{19}$ There is much controversy about whether packing actually reduces this risk. Bugten et al., ${ }^{20}$ described significantly increased synechiae in those treated with no packing when compared with those who were packed with a non-absorbable nasal pack $(\mathrm{p}<0.001)$ However, when Gelfoam was compared to no nasal packing in a study of 21 patients undergoing FESS with or without septoplasty, no significant difference was observed in synechia formation, granulation tissue or the presence of pus, or edema within the first four months following surgery. Again, the power of this study was likely to be low given the low number of patients The longer raw mucosal surfaces are in contact with biomaterials may also play a role. ${ }^{2}$ In animal models, longer exposure to biomaterials resulted in inflammatory reactions and incorporation of the foreign material into the mucosa. ${ }^{21,22}$ The exposure of mucosa to SPF is relatively short, it degrading enough after two days to facilitate removal via suction and would thus potentiate less synechiae. However, in one study SPF and Merocel were compared at week 4 post-FESS No patients demonstrated adhesion formation although this may have been a consequence of less extensive surgery as turbinoplasties and turbinectomies were excluded.$^{2}$ Another hypothesis is that agents which stimulate the intrinsic coagulation pathway increase mucosal inflammation and hence synechia. ${ }^{23,24}$ Thus, haemostatic nasal gels would be expected to increase synechia formation. This was not found to be the case in a single arm study designed to demonstrate the effectiveness of Surgiflo. In this study, none of the 30 patients with a bleeding surface post-FESS produced adhesions following packing. Similarly, in another study comparing the use of Floseal and a neuropatty middle meatal spacer with a neuropatty alone, a significant reduction in crusting after one week $(2.4 \% \mathrm{cf} 18.6 \% \mathrm{P}=0.015)$ was demonstrated when Floseal was added. This effect was lost, however, upon further follow-up at 1 and 3 months. ${ }^{8}$ Other studies have demonstrated an increase in granulation and synechiae in Floseal when compared with thrombin-soaked gelatin foam. ${ }^{25,26}$ It has been hypothesised that this is a consequence of a slower absorption profile of Floseal which takes 6 to 8 weeks to dissolve. ${ }^{8,27}$

If packing is to be undertaken in an attempt to reduce synechiae, further controversy exists regarding whether absorbable or nonabsorbable packs are superior. In one study, Merogel was compared to PVA sponge and showed less adhesions at 12 weeks ( $4.6 \%$ cf $29.7 \%$ $\mathrm{p}<0.001$ ). To support this finding $36.4 \%$ of those treated with Merogel had no granulation tissue at 4 weeks when compared to $10.5 \%$ in the Merogel group $\mathrm{p}<0.001 .{ }^{19}$ However, in a randomised controlled trial Miller et al., ${ }^{4}$ found no increase in adhesion formation with Merogel when compared to Merocel. ${ }^{4}$ In a further study, Nasopore was compared to Merocel and petroleum jelly impregnated gauze in a retrospective study of 626 patients undergoing FESS and no significant differences in the incidence of synechia among the three $(10.5 \%, 8.8 \%$ and $8 \%$ respectively) within 3 to 4 weeks $(\mathrm{p}=0.584)$. Finally, when Nasopore was compared to a traditional middle meatal spacer (Merocel in a glove finger) increased inflammation was observed at 4 weeks $(\mathrm{p}=0.03)$ but this effect was lost by week 12 $(\mathrm{p}=1.00)$. It was suggested that Nasopore produced slower healing initially than compared with a traditional middle meatal spacer. ${ }^{13}$ Our data demonstrated a low rate of synechiae with Nasopore $(1 \%)$ in a group which was largely treated with extensive surgery and thus at greater risk.
Finally, with respect to Nasopore facilitating FESS being performed as a day-case procedure there is little information in the literature and hospital stay is not a typically measured outcome. Some papers have provided information regarding hospital stay in their study protocol. Jameson et al discharged patients on the same day of surgery with either Floseal or a neuropatty middle meatal spacer. They observed no complications in either. Wee et al utilised absorbable Gelfoam in one nostril and no packing in the other in the same patients and discharged them after one day observing no acute complications. Kim et al in 2011 discuss removing both Merocel and SPF packing on the $2^{\text {nd }}$ postoperative day and discharging them. ${ }^{2}$ Antisdel et al., ${ }^{21}$ also discharged patients on the $2^{\text {nd }}$ postoperative day after utilising MPH (a haemostatic powder) to treat the nose after sinus surgery. In our cohort, $49 \%$ of patients went home on the day of surgery and $51 \%$ remained in hospital overnight to be discharged the following day. Distance from the hospital was not a factor in increasing patient stay assuming they stayed on the mainland. However, patients requiring a flight to a peripheral island showed an increased duration of stay. Those who had surgery in the afternoon increased their chance of staying overnight by $20 \%$.

\section{Conclusions}

Based on our data, it would appear that Nasopore is safe and efficient and results in a minimum of post-operative complications. As this nasal packing does not need removal, the patients can safely be sent home with the Nasopore in situ. In addition, the discomfort associated with the removal of permanent packing is avoided, thus helping to improve the overall experience of the patient undergoing FESS with or without additional procedures.

\section{Acknowledgments}

None.

\section{Conflicts of interest}

The authors declare that there is no conflicts of interest.

\section{References}

1. Hastan D, Fokkens WJ, Bachert $\mathrm{C}$, et al. Chronic rhinosinusitis in Europe--an underestimated disease. A GA ${ }^{2} \mathrm{LEN}$ study. Allergy. 2011;66(9):1216-1223

2. Kim YS, Kim YH, Kim NH, et al. A prospective, randomized, singleblinded controlled trial on biodegradable synthetic polyurethane foam as a packing material after septoplasty. Am J Rhinol Allergy. 2011;25(2):e77-e79.

3. Weber RK. Nasal packing and stenting. GMS Curr Top Otorhinolaryngol Head Neck Surg. 2009;8: Doc02.

4. Miller RS, Steward DL, Tami TA, et al. The clinical effects of hyaluronic acid ester nasal dressing (Merogel) on intranasal wound healing after functional endoscopic sinus surgery. Otolaryngol Head Neck Surg. 2003;128(6):862-869.

5. Eliashar R, Gross M, Wohlgelernter J, et al. Packing in endoscopic sinus surgery: is it really required? Otolaryngol Head Neck Surg. 2006;134(2):276-279.

6. Athanasiadis T, Beule AG, Wormald PJ. Effects of topical anti fibrinolytics in endoscopic sinus surgery: a pilot randomized controlled trial. Am J Rhinol. 2007;21(6):737-742.

7. Wang YP, Wang MC, Chen YC, et al. The effects of Vaseline gauze strip, Merocel, and Nasopore on the formation of synechiae and excessive granulation tissue in the middle meatus and the incidence of major postoperative bleeding after endoscopic sinus surgery. J Chin Med Assoc. 2011;74(1):16-21. 
8. Jameson M, Gross CW, Kountakis SE. FloSeal use in endoscopic sinus surgery: effect on postoperative bleeding and synechiae formation. $\mathrm{Am} \mathrm{J}$ Otolaryngol. 2006;7(2):86-90.

9. Huggins S. Control of hemorrhage in otorhinolaryngologic surgery with oxidized regenerated cellulose. Eye Ear Nose Throat Mon. 1969;48(7):420-423.

10. Valentine R, Wormald PJ, Sindwani R. Advances in absorbable biomaterials and nasal packing. Otolaryngol Clin North Am. 2009;42(5):813-828.

11. Mehta U, Huber TC, Sindwani R. Patient expectations and recovery following endoscopic sinus surgery. Otolaryngol Head Neck Surg. 2006;134(3):483-487.

12. Antisdel JL, West-Denning JL, Sindwani R. Effect of microporous polysaccharide hemospheres (MPH) on bleeding after endoscopic sinus surgery: randomized controlled study. Otolaryngol Head Neck Surg. 2009;141(3):353-357.

13. Shoman N, Gheriani H, Flamer D, et al. Prospective, double-blind, randomized trial evaluating patient satisfaction, bleeding, and wound healing using biodegradable synthetic polyurethane foam (NasoPore) as a middle meatal spacer in functional endoscopic sinus surgery. $J$ Otolaryngol Head Neck Surg. 2009;38(1):112-118.

14. Orlandi RR, Lanza DC. Is nasal packing necessary following endoscopic sinus surgery? Laryngoscope. 2004;114(9):1541-1544.

15. Wee JH, Lee CH, Rhee CS, et al. Comparison between Gelfoam packing and no packing after endoscopic sinus surgery in the same patients. Eur Arch Otorhinolaryngol. 2012;269(3):897-903.

16. Stankiewicz JA. Complications of endoscopic sinus surgery. Otolaryngol Clin North Am. 1989;22(4):749-758.

17. Baumann A, Caversaccio M. Hemostasis in endoscopic sinus surgery using a specific gelatin-thrombin based agent (FloSeal). Rhinology. 2003;41(4):244-249.
18. Catalano PJ, Roffman EJ. Evaluation of middle meatal stenting after minimally invasive sinus techniques (MIST). Otolaryngol Head Neck Surg. 2003;128(6):875-881.

19. Berlucchi M, Castelnuovo P, Vincenzi A, et al. Endoscopic outcomes of resorbable nasal packing after functional endoscopic sinus surgery: a multicenter prospective randomized controlled study. Eur Arch Otorhinolaryngol. 2009;266(6):839-845.

20. Bugten V, Nordgard S, Skogvoll E, et al. Effects of nonabsorbable packing in middle meatus after sinus surgery. Laryngoscope. 2006;116(1):83-88.

21. Antisdel JL, Janney CG, Long JP, et al. Hemostatic agent microporous polysaccharide hemospheres (MPH) does not affect healing or intact sinus mucosa. Laryngoscope. 2008;118(7):1265-1269.

22. Maccabee MS, Trune DR, Hwang PH. Effects of topically applied biomaterials on paranasal sinus mucosal healing. Am $J$ Rhinol. 2003;17(4):203-207.

23. Levi M, van der Poll T. Two-way interactions between inflammation and coagulation. Trends Cardiovasc Med. 2005;15(7): 254-259.

24. Levi $M$, van der Poll T, Buller HR. Bidirectional relation between inflammation and coagulation. Circulation. 2004;109(22):2698-2704.

25. Chandra RK, Conley DB, Haines GK 3rd, Kern RC. Long-term effects of FloSeal packing after endoscopic sinus surgery. Am J Rhinol. $2005 ; 19(3): 240-243$

26. Chandra RK, Conley DB, Kern RC. The effect of FloSeal on mucosal healing after endoscopic sinus surgery: a comparison with thrombinsoaked gelatin foam. Am J Rhinol. 2003;17(1):51-55.

27. Oz MC, Cosgrove DM, Badduke BR, et al. Controlled clinical trial of a novel hemostatic agent in cardiac surgery. The Fusion Matrix Study Group. Ann Thorac Surg. 2000;69(5):1376-1382. 